\title{
Magnetic and magnetocaloric properties of as-cast $\mathrm{Gd}_{2}$ In
}

\author{
S. V. Taskaev ${ }^{\dagger, 1,2,3}$, V. V. Khovaylo ${ }^{2,3}$, M. N. Ulyanov ${ }^{1}$, D. S. Bataev ${ }^{1}$, A. A. Basharova ${ }^{1}$, \\ M. V. Kononova ${ }^{1,3}$, D. V. Plakhotskiy ${ }^{1}$, M. Yu. Bogush ${ }^{1}$, D. A. Zherebtsov ${ }^{2}$ \\ †tsv@csu.ru
}

\author{
${ }^{1}$ Chelyabinsk State University, Chelyabinsk, 454001, Russia \\ ${ }^{2}$ National Research South Ural State University, Chelyabinsk, 454080, Russia \\ ${ }^{3}$ National University of Science and Technology “MISIS”, Moscow, 119049, Russia
}

\begin{abstract}
This paper presents results of experimental studies of magnetic and magnetocaloric properties of as-cast polycrystalline samples of a $\mathrm{Gd}_{2}$ In intermetallic compound, which crystallizes into a hexagonal structure of the $\mathrm{Ni}_{2} \mathrm{In}$ type. Measurements of the field and temperature dependences of the magnetization carried out in a temperature range from 50 to $300 \mathrm{~K}$ and in magnetic fields up to $3 \mathrm{~T}$ showed that the as-cast $\mathrm{Gd}_{2} \mathrm{In}$ samples undergo ferromagnetic ordering upon cooling below Curie temperature $T_{C}=194 \mathrm{~K}$. With further cooling, a metamagnetic transition from the ferromagnetic into an antiferromagnetic phase is observed at a temperature $T_{m}=95 \mathrm{~K}$, which is accompanied by pronounced anomalies in the temperature and field dependences of the magnetization. An external magnetic field stabilizes the ferromagnetic phase, shifting $T_{m}$ toward low temperatures. The isothermal magnetic entropy change in the $\Delta S_{m}$, calculated using the Maxwell relation, shows a peak at the Curie temperature, which, when the magnetic field changes from 0 to $3 \mathrm{~T}$, reaches its maximum value $\Delta S_{m}=-3.25 \mathrm{~J} / \mathrm{kg} \cdot \mathrm{K}$. The temperature dependence of $\Delta S_{m}$ clearly indicates a crossover of the magnetocaloric effect from direct to inverse one, which occurs in the vicinity of the metamagnetic transition. The experimental data obtained in our work on unannealed $\mathrm{Gd}_{2} \mathrm{In}$ samples are in a good qualitative and quantitative agreement with the data published for the annealed $\mathrm{Gd}_{2}$ In samples. Thus, the results of this work indicate that the attainment of a homogeneous state in polycrystalline $\mathrm{Gd}_{2}$ In samples, which can affect their magnetic properties, can occur during cooling of the as-cast ingot on a cold crucible and does not require an additional heat treatment.
\end{abstract}

Keywords: magnetocaloric effect, metamagnetic transition, Curie temperature, gadolinium alloys.

\section{Магнитные и магнитокалорические свойства литого Gd $\mathbf{I n}$}

\author{
Таскаев С. В. ${ }^{\dagger, 1,2,3}$, Ховайло В. В. ${ }^{2,3}$, Ульянов М. Н. ${ }^{1}$, Батаев Д. С. ${ }^{1}$, Башарова А. А. ${ }^{1}$, \\ Кононова М. В. ${ }^{1,3}$, Плахотский Д. В. ${ }^{1}$, Богуш М. Ю. ${ }^{1}$, Жеребцов Д. А. ${ }^{2}$ \\ ${ }^{1}$ Челябинский государственный университет, Челябинск, 454001, Россия \\ ${ }^{2}$ Южно-Уральский государственный университет (национальный исследовательский университет), \\ Челябинск, 454080, Россия \\ ${ }^{3}$ Национальный исследовательский технологический университет «МИСиС», Москва, 119049, Россия
}

В данной работе представлены результаты экспериментальных исследований магнитных и магнитокалорических свойств литых поликристаллических образцов интерметаллического соединения $\mathrm{Gd}_{2} \mathrm{In}$, которое кристаллизуется в гексагональную структуру типа $\mathrm{Ni}_{2}$ In. Измерения полевых и температурных зависимостей намагниченности, выполненных в температурном интервале от 50 до 300 К и в магнитных полях до 3 Тл показали, что литые образцы $\mathrm{Gd}_{2} \mathrm{In}$ претерпевают ферромагнитное упорядочение при охлаждении ниже температуры Кюри $T_{C}=194 \mathrm{~K}$. При дальнейшем охлаждении наблюдается метамагнитный переход из ферромагнитной в антиферромагнитную фазу при температуре $T_{m}=95 \mathrm{~K}$, что сопровождается ярко выраженными аномалиями на температурных и полевых зависимостях намагниченности. Внешнее магнитное поле стабилизирует ферромагнитную фазу, смещая $T_{m}$ в область низких температур. Изотермическое изменение магнитной энтропии $\Delta S_{m}$, вычисленное при помощи соотношения 
Максвелла, демонстрирует пик при температуре Кюри, который при изменении магнитного поля от 0 до 3 Тл достигает максимального значения $\Delta S_{m}=-3.25$ Дж/кг·К. Зависимость $\Delta S_{m}$ от температуры ясно указывает на кроссовер магнитокалорического эффекта от прямого до инверсного, который происходит в окрестности метамагнитного перехода. Экспериментальные данные, полученные в нашей работе на неотожженных образцах $\mathrm{Gd}_{2} \mathrm{In}$, находятся в хорошем качественном и количественном согласии с данными, опубликованными для отожженных образцов $\mathrm{Gd}_{2} \mathrm{In}$. Таким образом, результаты данной работы указывают на то, что достижение гомогенного состояния в поликристаллических образцах $\mathrm{Gd}_{2} \mathrm{In}$, которой может влиять на их магнитные свойства, может происходить в процессе охлаждения литого слитка на холодном поду и не требует дополнительной термообработки.

Ключевые слова: магнитокалорический эффект, метамагнитный переход, температура Кюри, сплавы гадолиния.

\section{1. Введение}

В последние годы идея использования магнитокалорического эффекта (МКЭ) в технологии ожижения природных и криогенных газов привлекает растущее внимание исследователей [1]. Этот интерес обусловлен с одной стороны низкой эффективностью традиционных методов ожижения газов при температурах ниже 150 К, а с другой стороны - существенным прогрессом в разработке сверхпроводящих магнитов, которые могут генерировать сильные магнитные поля, до 22 Тл. Принимая во внимание, что МКЭ, а именно изотермическое изменение магнитной энтропии $\Delta S_{m}$, в окрестности магнитных фазовых переходов 2-го рода зависит от приложенного магнитного поля как $-\Delta S_{m} \sim H^{2 / 3}$ [2], идея применения технологии магнитного охлаждения в сильных магнитных полях сверхпроводящих соленоидов для ожижения газов имеет большую практическую значимость. С материаловедческой точки зрения, для реализации этой идеи необходимо исследовать особенности магнитокалорического эффекта в сильных магнитных полях и отработать технологию получения магнитокалорических материалов.

Подавляющее большинство материалов, в которых наблюдается существенный МКЭ в криогенной области температур, представляют собой редкоземельные сплавы и соединения [3]. Большой магнитокалорический эффект в этих материалах обусловлен главным образом большим магнитным моментом этих соединений, в частности соединений на основе Gd. Так, исследования интерметаллидов $\mathrm{Gd}_{3} \mathrm{~T}(\mathrm{~T}-3 d$ или $4 d$ переходной металл) показали, что в $\mathrm{Gd}_{3} \mathrm{Co}, \mathrm{Gd}_{3} \mathrm{Ni}$ и $\mathrm{Gd}_{3} \mathrm{Rh}$ наблюдаются сравнительно небольшие величины МКЭ, что обусловлено доминированием антиферромагнитных взаимодействий в этих соединениях. Наиболее яркий $\mathrm{MКЭ,} \Delta S_{m}=-30$ Дж/кг·К при изменении магнитного поля на 5 Тл, был обнаружен в соединении $\mathrm{Gd}_{3} \mathrm{Ru}$, которое претерпевает магнитный фазовый переход 1-го рода при температуре Кюри $T_{C}=54$ K [4]. Рассматривая магнитокалорические свойства других соединений на основе $\mathrm{Gd}$, необходимо упомянуть фазу Лавеса $\mathrm{GdNi}_{2}$, где МКЭ исследовался в магнитных полях до 50 Тл [5], фазу Лавеса $\mathrm{GdAl}_{2}$, в которой ярко проявляется взаимосвязь между структурными и магнитными свойствами [6] и интерметаллическое соединение $\mathrm{Gd}_{2} \mathrm{In}$, в котором наблюдается метамагнитный переход при температурах 100 К [7], сопровождаемый инверсией знака МКЭ [8].
Принимая во внимание перспективы практических применений этих материалов, необходимо, наряду с магнитокалорическими свойствами, исследовать также зависимость этих свойств от метода получения и режимов термообработки экспериментальных образцов. Как правило, поликристаллические образцы редкоземельных соединений получают методом дуговой или индукционной плавки. После этого образцы отжигаются в вакууме или атмосфере аргона при высоких температурах в течении длительного времени; причем выбор параметров термообработки как правило не обосновывается. Так, в случае соединения $\mathrm{Gd}_{2} \mathrm{In}$ температура отжига варьировалась от 973 К $[8,9]$ до 1073 К [10], а сам отжиг занимал время от 2 до 5 дней $[8,9,11]$. Насколько нам известно, ни для $\mathrm{Gd}_{2} \mathrm{In}$, ни для других бинарных соединений гадолиния вопросы взаимосвязи режимов термообработки и магнитных свойств в литературе не освещались. Принимая во внимание, что термообработка является затратной процедурой как с точки зрения ресурсов, так и времени, в данной работе были выполнены исследования магнитных свойств неотожженных образцов $\mathrm{Gd}_{2} \mathrm{In}$ и сравнение этих свойств с доступными в литературе данными, полученными на подвергнутых термообработке образцах этого соединения.

\section{2. Детали эксперимента}

Поликристаллический слиток $\mathrm{Gd}_{2} \mathrm{In}$ был получен методом дуговой плавки на водоохлаждаемом медном поду в защитной атмосфере чистого аргона с использованием неплавящегося вольфрамового электрода из исходных высокочистых химических элементов Gd (чистота 99.98 ат.\%) и In (чистота 99.998 ат.\%). Для достижения однородности по химическому составу слиток переплавлялся трижды. После выключения дуги литой слиток остывал в камере дуговой печи. Никакой дополнительной термообработки впоследствии не проводилось.

Рентгеноструктурный и рентгенофазовый анализ проводился при комнатной температуре на рентгеновском дифрактометре Rigaku Ultima IV с использованием $\mathrm{Cu}_{\mathrm{Ka}}$-излучения. Элементный состав литого слитка исследовался на энергодисперсионном рентгенофлуоресцентном спектрометре ARL QUANT'X и растровом электронном микроскопе JEOL JSM-6510LA. Bсе магнитные измерения проводились с использованием 
магнитометра Versa Lab компании Quantum Design в соответствии со стандартными процедурами измерения при температурах от 50 до 300 К и в магнитных полях до 3 Тл.

\section{3. Результаты и обсуждение}

На Рис. 1 представлена дифрактограмма $\mathrm{Gd}_{2} \mathrm{In}$, полученная при комнатной температуре. Кристаллическая структура исследуемого соединения является гексагональной (структурный тип $\mathrm{Ni}_{2} \mathrm{In}$ ) с параметрами решетки $a=b=0.5461 \mathrm{нм}, c=0.6771$ нм и $\alpha=\beta=90^{\circ}$, $\gamma=120^{\circ}$, которые находятся в хорошем согласии с параметрами решетки отожженных образцов $[7,10]$. Судя по рентгеновским данным (Рис. 1) литой образец $\mathrm{Gd}_{2} \mathrm{In}$ примесных фаз не содержит.

Температурные зависимости намагниченности $M(T)$ литого $\mathrm{Gd}_{2} \mathrm{In}$, измеренные при охлаждении в магнитных полях от 0.1 до 3 Тл с шагом 0.1 Тл представлены на Рис. 2. Видно, что в слабых магнитных полях на зависимостях $M(T)$ наблюдаются две аномалии, в окрестности 200 и 100 К, которые соответствуют установлению магнитного упорядочения и метамагнитному переходу, соответственно. При этом низкотемпературная аномалия постепенно размывается при увеличении напряженности внешнего магнитного поля и полностью исчезает в магнитных полях выше 2 Тл (Рис. 2). Определенная из зависимости $M(T)$, измеренной в магнитном поле 0.1 Тл, температура Кюри и температура метамагнитного перехода литого $\mathrm{Gd}_{2} \mathrm{In}$ была найдена равной $T_{C}=194$ К и $T_{m}=96 \mathrm{~K}$, соответственно. Эти значения хорошо согласуются с данными, полученными для отожженных поликристаллических образцов $\left(T_{C} \approx 187 \mathrm{~K}\right.$ и $\left.T_{m} \approx 100 \mathrm{~K}\right)[7,9]$.

Говоря о природе матамагнитного перехода, который наблюдается в нашем образце при $T_{m}=96 \mathrm{~K}$, в настоящее время предполагается, что метамагнитная аномалия соответствует переходу из ферромагнитной в антиферромагнитную фазу. Отметим, что детальная картина упорядочения спинов в низкотемпературной антиферромагнитной и высокотемпературной ферромагнитной структуре остается невыясненной до наших дней из-за сильного поглощения нейтронов гадолинием, что не позволяет применять для Gd-содержащих соединений метод установления магнитной структуры с помощью дифракции нейтронов. Хотя ферромагнитное упорядочение спинов в высокотемпературной магнитной фазе $\mathrm{Gd}_{2} \mathrm{In}$ не вызывает сомнений у большинства исследователей, в ряде работ было предположено [12,13], что конфигурация спинов в низкотемпературной магнитной фазе скорее всего не является коллинеарной.

Как уже отмечалось выше, и как следует из Рис. 2, температура метамагнитного перехода $T_{m}$ быстро понижается при увеличении напряженности внешнего магнитного поля. Приведенная в литературе оценка чувствительности $T_{m}$ ко внешнему магнитному полю в отожженном $\mathrm{Gd}_{2} \mathrm{In}$ дает значение порядка $97 \mathrm{~K} / \mathrm{T}$ [11]. В случае литого $\mathrm{Gd}_{2} \mathrm{In}$ полевая зависимость намагниченности $M(H)$, измеренная при $T=50$ К (Рис. 3) демонстрирует ярко выраженное изменение характера кривой

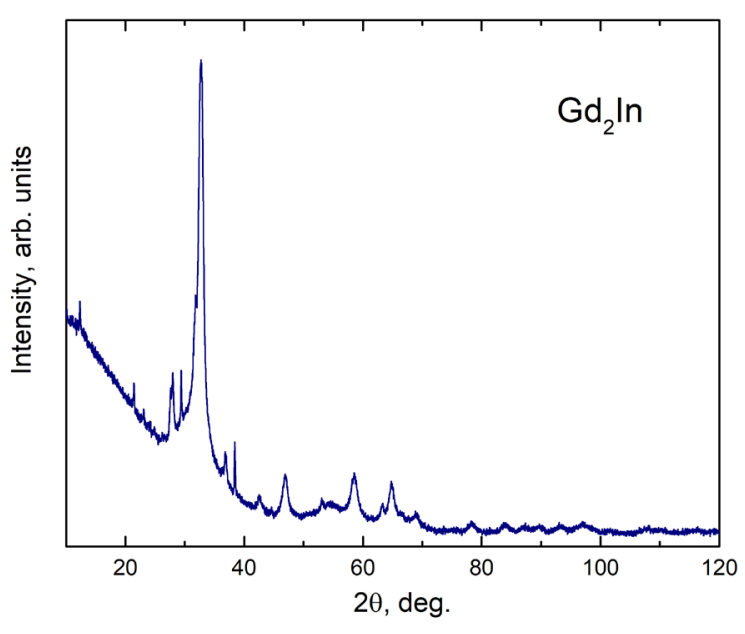

Рис. 1. Рентгенограмма образца $\mathrm{Gd}_{2} \mathrm{In}$, снятая при комнатной температуре.

Fig. 1. X-ray pattern taken from $\mathrm{Gd}_{2} \mathrm{In}$ sample at room temperature.

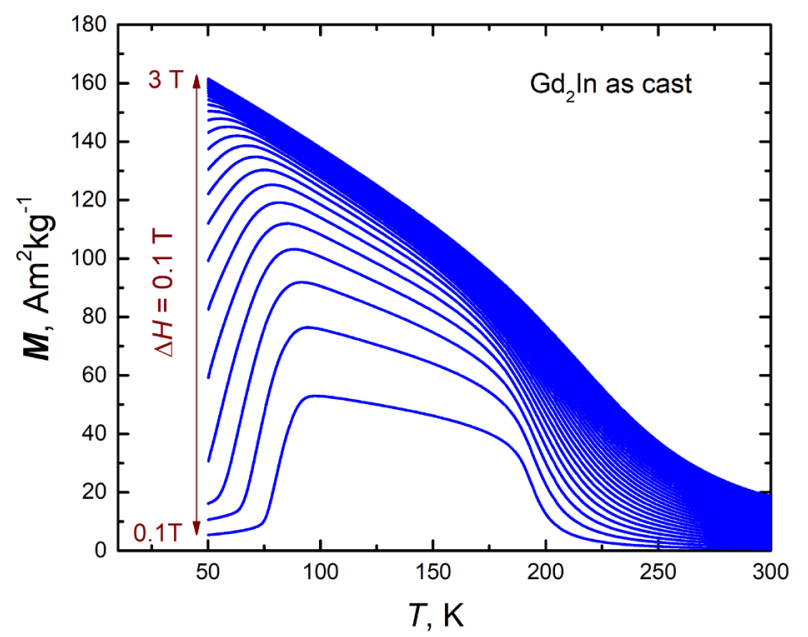

Рис. 2. Температурные зависимости литого $\mathrm{Gd}_{2} \mathrm{In}$, измеренные в магнитных полях от 0.1 до 3 Тл.

Fig. 2. Temperature dependences of magnetization for as-cast $\mathrm{Gd}_{2} \mathrm{In}$, measured in magnetic fields from 0.1 to $3 \mathrm{~T}$.

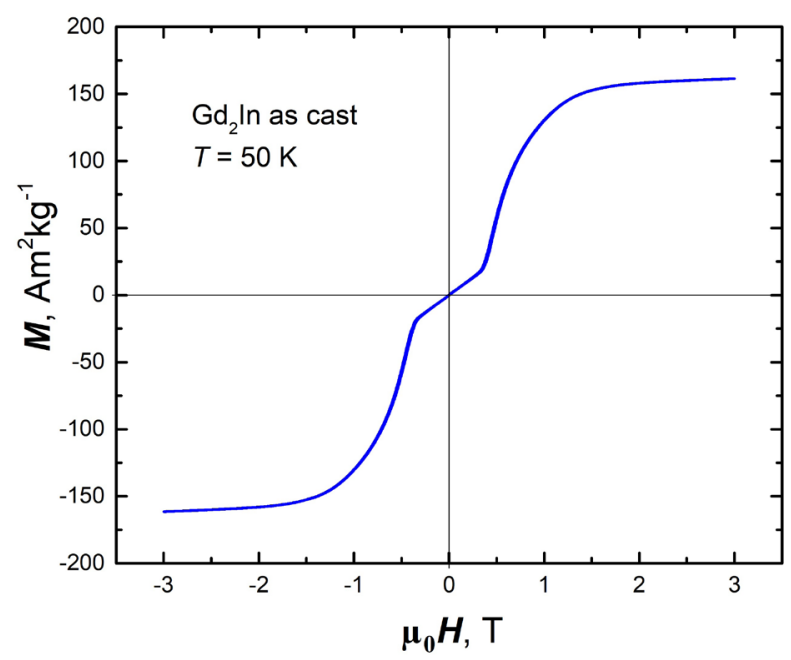

Рис. 3. Петля гистерезиса литого $\mathrm{Gd}_{2} \mathrm{In}$, измеренная при $T=50 \mathrm{~K}$. Fig. 3. Hysteresis loop of the as-cast $\mathrm{Gd}_{2} \mathrm{In}$, measured at $T=50 \mathrm{~K}$. 
от типичного для антиферромагнетиков линейного хода до быстрого роста $M$ при достижении критической величины магнитного поля, равной 0.34 Тл (Рис. 3). Очевидно, что изменение хода кривой намагниченности при $H=0.34$ Тл соответствует магнитоиндуцированному переходу от антиферромагнитного к ферромагнитному упорядочению. Таким образом, грубая оценка чувствительности метамагнитного перехода к внешнему магнитному полю в литом $\mathrm{Gd}_{2} \mathrm{In}$ дает величину $135 \mathrm{~K} / \mathrm{Tл}$. Определенная методом экстраполяции намагниченность насыщения литого Gd In при $T=50$ К (Рис. 3) была найдена равной $154 \mathrm{~A} \cdot \mathrm{M}^{2} / \mathrm{\kappa}$, что хорошо согласуется в данными для отожженных образцов [8].

Изотермическое изменение магнитной энтропии $\Delta S_{m}$, вычисленной из данных экспериментальных измерений намагниченности при помощи соотношения Максвелла, показано на Рис. 4. Можно отметить следующие характерные особенности температурных зависимостей $\Delta S_{m}$. Во-первых, $\Delta S_{m}$ демонстрирует четкий пик при $T=196 \mathrm{~K}$, что практически совпадает с температурой Кюри этого образца $T_{C}=194$ К. Величина этого пика возрастает при росте величины изменения приложенного магнитного поля и достигает значения $\Delta S_{m}=-3.25$ Дж/кг $\cdot$ К при изменении магнитного поля $\Delta H=3$ Тл (Рис. 4). Это значение практически совпадает с величиной изотермического изменения магнитной энтропии $\left(\Delta S_{m}=-3.5\right.$ Дж/кг $\cdot$ К), которое сообщалось для отожженных образцов $\mathrm{Gd}_{2} \mathrm{In}[8,11]$ и допированного алюминием сплава $\mathrm{Gd}_{2} \mathrm{In}_{0.8} \mathrm{Al}_{0.2}$ [14]. При температурах ниже 100 К, где наблюдается метамагнитный переход, происходит смена знака МКЭ. Такое поведение $\Delta S_{m}$ определяется тем фактом, что в окрестности метамагнитного перехода высокотемпературная ферромагнитная фаза является более упорядоченной, чем низкотемпературная антиферромагнитная фаза. Подобное поведение сообщалось и для других систем, претерпевающих метатагнитные переходы из более упорядоченной в менее упорядоченную фазу, таких как соединение FeRh [15], сплав Гейслера Ni-Mn-Sn [16] и др.

\section{4. Заключение}

В данной работе представлены результаты экспериментальных исследований магнитных и магнитокалорических свойств литого интерметаллического соединения $\mathrm{Gd}_{2} \mathrm{In}$. Наиболее интересным и значимым результатом нашей работы является то, что магнитные свойства и характеристики магнитокалорического эффекта изученных в нашей работе неотожженных образцов $\mathrm{Gd}_{2} \mathrm{In}$ ничем не уступают образцам, подвергнутым тщательной и долговременной термообработке. Представляется вероятным, что идентичность магнитных свойств отожженных и неотожженных образцов обусловлено тем фактом, что $\mathrm{Gd}_{2} \mathrm{In}$ является упорядоченным интерметаллическим соединением, область существование которого на двойной фазовой диаграмме Gd-In простирается от комнатных температур вплоть до температуры плавления 1453 K [17]. Таким образом, используемый рядом исследователей отжиг и закалка от высоких температур порядка 900-1100 К

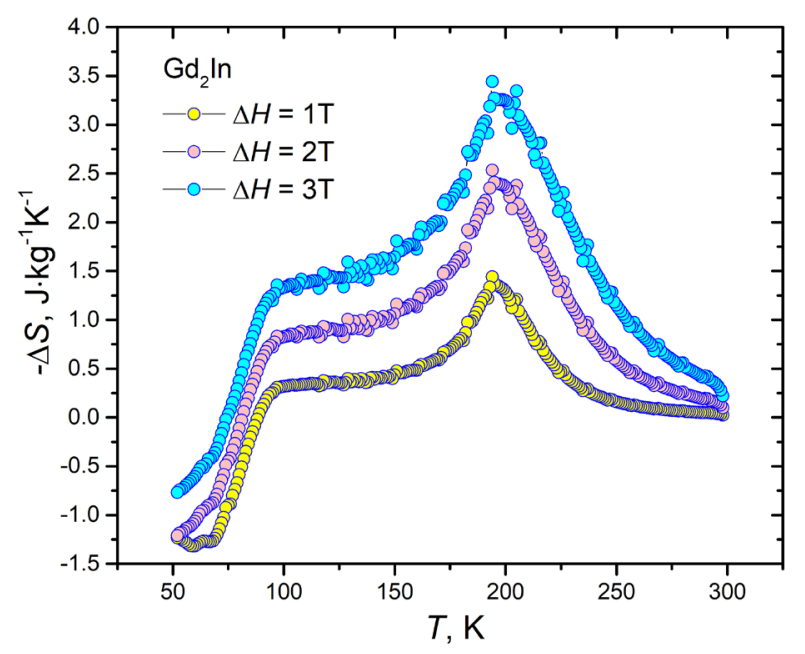

Pис. 4. (Color online) Изотермическое изменение магнитной энтропии $\Delta S_{m}$ в литом $\mathrm{Gd}_{2}$ In при изменении магнитного поля на 1, 2 и 3 Тл.

Fig. 4. (Color online) Isothermal magnetic entropy change $\Delta S_{m}$ in the as-cast $\mathrm{Gd}_{2} \mathrm{In}$ for the magnetic field change 1, 2, and $3 \mathrm{~T}$.

не приводит к существенному улучшению структурного упорядочения по сравнению с литыми образцами, которые не прошли термообработку. Более того, некоторое расширение области стабильности $\mathrm{Gd}_{2} \mathrm{In}$ при высоких температурах [17] может приводить к формированию дефектов упаковки при закалке образцов с нестехиометрическим содержанием In. Наряду с этим, полученные нами результаты указывают на то, что в некоторых случаях представляется возможным избежать затратной процедуры термообработки редкоземельных интерметаллидов, которые в будущем могут найти практическое применение в качестве рабочего тела для работающих на МКЭ устройств для ожижения криогенных газов.

Благодарности/Acknowledgements. Работа выполнена при поддержке Российского научного фонда (грант № 18-42-06201). / This work was supported by the Russian Science Foundation (Grant No. 18-42-06201).

\section{Литература/References}

1. H. Zhang, R. Gimaev, B. Kovalev, K. Kamilov, V. Zverev, A. Tishin. Physica B. 558, 65 (2019). Crossref

2. H. Oesterreicher, F.T. Parker. J. Appl. Phys. 55, 4334 (1984). $\underline{\text { Crossref }}$

3. A.S. Andreenko, K.P. Belov, S. A. Nikitin, A. M. Tishin. Physics - Uspekhi. 32, 649 (1989). Crossref

4. J. C. B. Monteiro, R. D. dos Reis, F. G. Gandra. Appl. Phys. Lett. 106, 194106 (2015). Crossref

5. S. Taskaev, V. Khovaylo, K. Skokov, W. Liu, E. Bykov, M. Ulyanov, D. Bataev, A. Basharova, M. Kononova, D. Plakhotskiy, M. Bogush, T. Gottschall, O. Gutfleisch. J. Appl. Phys. 127, 233906 (2020). Crossref

6. V. G. de Paula, L. M. da Silva, A. O. dos Santos, R. Lang, L. Otubo, A. A. Coelho, L. P. Cardoso. Phys. Rev. B. 93, 094427 (2016). $\underline{\text { Crossref }}$ 
7. S. P. McAlister. J. Phys. F: Met. Phys. 14, 2167 (1984). Crossref

8. A. Bhattacharyya, S. Giri, S. Majumdar. J. Magn. Magn. Mater. 324, 1239 (2012). Crossref

9. V. Singh, A. Bhattacharyya, S. Majumdar, I. Dasgupta. J. Appl. Phys. 111, 053709 (2012). Crossref

10. W. Baela, A. Szytula. J. Less-Comm. Met. 138, 123 (1988). Crossref

11. Y. Yang, Y. Xie, X. Zhou, H. Zhong, Q. Jiang, S. Ma, Z. Zhong, W. Cui, Q. Wang. AIP Adv. 8, 056406 (2018). Crossref

12. C.-S. Jee, C.L. Lin, T. Mihalisin, X.-Q. Wang. J. Appl. Phys. 79, 5403 (1996). $\underline{\text { Crossref }}$

13. P.A. Stampe, X.Z. Zhou, H.P. Kunkel, J.A. Cowen,
G. Williams. J. Phys.: Condens. Matter. 9, 3763 (1997).

Crossref

14. S. Tencé, B. Chevalier. J. Magn. Magn. Mater. 399, 46 (2016). Crossref

15. E. Stern-Taulats, A. Planes, P. Lloveras, M. Barrio, J.-L. Tamarit, S. Pramanick, S. Majumdar, C. Frontera, L. Mañosa. Phys. Rev. B. 89, 214105 (2014). Crossref

16. V.V. Khovaylo, K.P. Skokov, O. Gutfleisch, H. Miki, T. Takagi, T. Kanomata, V.V. Koledov, V.G. Shavrov, G. Wang, E. Palacios, J. Bartolomé, R. Burriel. Phys. Rev. B. 81, 214406 (2010). $\underline{\text { Crossref }}$

17. A. Palenzona, S. Cirafici. Bulletin of Alloy Phase Diagrams. 10, 234 (1989). $\underline{\text { Crossref }}$ 